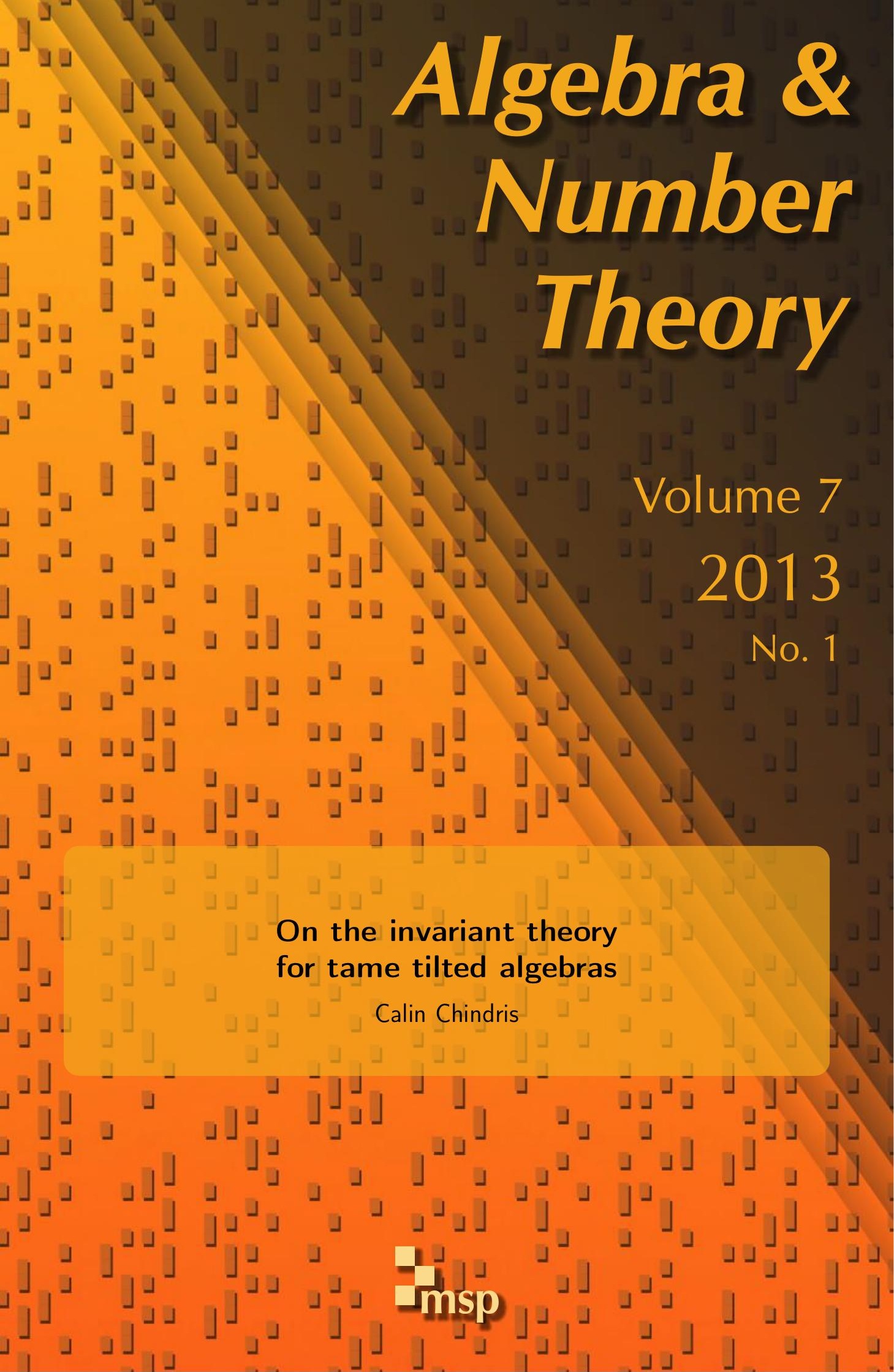




\title{
On the invariant theory for tame tilted algebras
}

\author{
Calin Chindris
}

\begin{abstract}
We show that a tilted algebra $A$ is tame if and only if for each generic root $\boldsymbol{d}$ of $A$ and each indecomposable irreducible component $C$ of $\bmod (A, \boldsymbol{d})$, the field of rational invariants $k(C)^{\mathrm{GL}(d)}$ is isomorphic to $k$ or $k(x)$. Next, we show that the tame tilted algebras are precisely those tilted algebras $A$ with the property that for each generic root $\boldsymbol{d}$ of $A$ and each indecomposable irreducible component $C \subseteq \bmod (A, \boldsymbol{d})$, the moduli space $\mathcal{M}(C)_{\theta}^{s s}$ is either a point or just $\mathbb{P}^{1}$ whenever $\theta$ is an integral weight for which $C_{\theta}^{s} \neq \varnothing$. We furthermore show that the tameness of a tilted algebra is equivalent to the moduli space $M(C)_{\theta}^{s s}$ being smooth for each generic root $\boldsymbol{d}$ of $A$, each indecomposable irreducible component $C \subseteq \bmod (A, \boldsymbol{d})$, and each integral weight $\theta$ for which $C_{\theta}^{s} \neq \varnothing$. As a consequence of this latter description, we show that the smoothness of the various moduli spaces of modules for a strongly simply connected algebra $A$ implies the tameness of $A$.

Along the way, we explain how moduli spaces of modules for finite-dimensional algebras behave with respect to tilting functors, and to theta-stable decompositions.
\end{abstract}

\section{Introduction}

Throughout this paper, we work over an algebraically closed field $k$ of characteristic zero. All algebras (associative and with identity) are assumed to be finitedimensional over $k$, and all modules are assumed to be finite-dimensional left modules.

One of the fundamental problems in the representation theory of algebras is that of classifying the indecomposable modules. Based on the complexity of the indecomposable modules, one distinguishes the class of tame algebras and that of wild algebras. According to the remarkable Tame-Wild Dichotomy Theorem of Drozd [1979], these two classes of algebras are disjoint and they cover the whole class of

The author was partially supported by NSF grant DMS- 1101383 .

MSC2010: primary 16G10; secondary 16R30, 16G60, 16G20.

Keywords: exceptional sequences, moduli spaces, rational invariants, tame and wild algebras, tilting. 
algebras. Since the representation theory of a wild algebra is at least as complicated as that of a free algebra in two variables, and since the latter theory is known to be undecidable, one can hope to meaningfully classify the indecomposable modules only for tame algebras. For more precise definitions, see [Simson and Skowroński 2007, Chapter XIX] and the reference therein.

An interesting task in the representation theory of algebras is to study the geometry of affine varieties of modules of fixed dimension vectors and the actions of the corresponding products of general linear groups associated to a given finitedimensional algebra $A$ over $k$. In particular, it would be interesting to find characterizations of prominent classes of tame algebras via geometric properties of their module varieties. This research direction has attracted much attention during the last two decades; see for example [Bobiński 2008; Bobiński and Skowroński 1999a; 1999b; 2002; Geiss and Schröer 2003; Riedtmann 2004; Riedtmann and Zwara 2004; 2008; Skowroński and Weyman 2000].

In this paper, we seek for characterizations of tame algebras in terms of invariant theory. A first result in this direction was obtained by Skowroński and Weyman [2000, Theorem 1], who showed that a finite-dimensional algebra of global dimension one is tame if and only if all of its algebras of semiinvariants are complete intersections. Unfortunately, this result does not extend to algebras of higher global dimension (not even of global dimension two), as shown by Kraśkiewicz [2001]. As was suggested by Weyman, in order to characterize the tameness of an algebra via invariant theory, one should impose geometric conditions on the various moduli spaces of semistable modules rather than on the entire algebras of semiinvariants.

In [Chindris 2011], the author has found a description of the tameness of path algebras and of canonical algebras in terms of the invariant theory of the algebras in question; see also [Domokos 2011]. In this paper, we continue this line of inquiry for the class of tilted algebras. Recall that a tilted algebra is an algebra of the form $\operatorname{End}_{H}(T)$, where $H$ is a connected finite-dimensional hereditary algebra and $T$ is a multiplicity-free tilting $H$-module, that is, $\operatorname{Ext}_{H}^{1}(T, T)=0$ and $T$ is the direct sum of $n$ pairwise nonisomorphic indecomposable modules with $n$ the rank of the Grothendieck group $K_{0}(H)$ of $H$. It has been proved by Kerner [1989, Theorem 6.2] that a tilted algebra $A$ is tame if and only if its Tits quadratic form $q_{A}$ is weakly nonnegative (takes nonnegative values on nonnegative vectors).

Theorem 1.1. Let $A$ be a tilted algebra. Then the following conditions are equivalent:

(1) A is tame;

(2) for each generic root $\boldsymbol{d}$ of $A$ and each indecomposable irreducible component $C$ of $\bmod (A, \boldsymbol{d})$, we have $k(C)^{\mathrm{GL}(\boldsymbol{d})} \simeq k$ or $k(x)$; 
(3) for each generic root $\boldsymbol{d}$ of $A$ and each indecomposable irreducible component $C \subseteq \bmod (A, \boldsymbol{d})$, the moduli space $\mathcal{M}(C)_{\theta}^{s s}$ is either a point or $\mathbb{P}^{1}$ whenever $\theta$ is an integral weight of $A$ for which $C_{\theta}^{s} \neq \varnothing$;

(4) for each generic root $\boldsymbol{d}$ of $A$ and each indecomposable irreducible component $C \subseteq \bmod (A, \boldsymbol{d})$, the moduli space $M(C)_{\theta}^{s s}$ is smooth whenever $\theta$ is an integral weight of $A$ for which $C_{\theta}^{s} \neq \varnothing$.

Following [Skowroński 1993], a triangular algebra $A$ is called strongly simply connected if the first Hochschild cohomology space $H H^{1}(C)$ of any convex subcategory $C$ of $A$ vanishes. It has been recently proved by Brüstle, de la Peña, and Skowroński [Brüstle et al. 2011, Main Theorem] that a strongly simply connected algebra $A$ is tame if and only if its Tits form $q_{A}$ is weakly nonnegative. As a consequence of Theorem 1.1 and another tameness criterion from [ibid., Corollary 1], we derive the following sufficient geometric criterion for the tameness of a strongly simply connected algebra:

Proposition 1.2. Let $A$ be a strongly simply connected algebra. Assume for each generic root d of $A$, each indecomposable irreducible component $C \subseteq \bmod (A, \boldsymbol{d})$, and each integral weight $\theta$ for which $C_{\theta}^{s} \neq \varnothing$, that $\mathcal{M}(C)_{\theta}^{s s}$ is a smooth variety. Then, $A$ is a tame algebra.

We would like to point out that the equivalence of (1) and (3) in Theorem 1.1 settles in the affirmative a conjecture of Weyman for the class of tilted algebras, while Proposition 1.2 proves one implication of Weyman's conjecture for the class of strongly simply connected algebras (for more details, see Remark 4).

Our next theorem, which is key in proving Theorem 1.1 and Proposition 1.2, identifies integral weights of an algebra for which the corresponding moduli spaces of semistable modules are preserved under titling. Our next theorem generalizes [Domokos and Lenzing 2000, Theorem 6.3 ] to arbitrary bound quiver algebras. (The details of our notation can be found in Section 3B.)

Theorem 1.3. Let $A=k Q / I$ be a bound quiver algebra, $T$ a basic tilting $A$ module, and $\theta$ an integral weight of $A$ that is well positioned with respect to $T$. Let $F$ be either the functor $\operatorname{Hom}_{A}\left(T,{ }_{-}\right)$, in case there are nonzero $\theta$-semistable torsion A-modules, or the functor $\operatorname{Ext}_{A}^{1}\left(T,{ }_{-}\right)$, in case there are nonzero $\theta$-semistable torsion-free A-modules. Denote the algebra $\operatorname{End}_{A}(T)^{\text {op }}$ by $B$ and let $u: K_{0}(A) \rightarrow$ $K_{0}(B)$ be the isometry induced by the tilting module T. Then,

(a) the functor $F$ defines an equivalence of categories between $\bmod (A)_{\theta}^{s s}$ and $\bmod (B)_{\theta^{\prime}}^{s s}$, where $\theta^{\prime}=\left|\theta \circ u^{-1}\right|$; and

(b) the bijective map $f: \mathcal{M}(A, \boldsymbol{d})_{\theta}^{s s} \rightarrow \mathcal{M}\left(B, \boldsymbol{d}^{\prime}\right)_{\theta^{\prime}}^{s s}$ induced by $F$ is an isomorphism of algebraic varieties, where $\boldsymbol{d}$ is a $\theta$-semistable dimension vector of $A$ and $\boldsymbol{d}^{\prime}=u(\boldsymbol{d})$ 
In particular, this theorem allows us to transfer much of the geometry of $A$ over to that of $B$; see for example Proposition 4.1.

It is natural to ask if the description of the fields of rational invariants and of the moduli spaces in Theorem 1.1 can be extended to irreducible components that are not necessarily indecomposable. To answer this question, we rely on two general reduction results. The first such result has been recently proved in [Chindris 2011, Proposition 4.7] and allows one to compute fields of rational invariants on irreducible components by reducing the considerations to the case where the irreducible components involved are indecomposable. For the second general reduction result, the starting point is Derksen and Weyman's notion [2011] of $\theta$-stable decomposition of representation spaces for quivers without oriented cycles. Here, we first extend their notion to irreducible components of module varieties, and then explain how to extend [Derksen and Weyman 2011, Theorem 3.20] to arbitrary bound quiver algebras:

Theorem 1.4. Let $A=k Q / I$ be a bound quiver algebra and let $C \subseteq \bmod (A, \boldsymbol{d})$ be a $\theta$-well-behaved irreducible component, where $\theta$ is an integral weight of $A$. Let

$$
C=m_{1} \cdot C_{1} \dot{+} \cdots \dot{+} m_{n} \cdot C_{n}
$$

be the $\theta$-stable decomposition of $C$, where $C_{i} \subseteq \bmod \left(A, \boldsymbol{d}_{i}\right)$ with $1 \leq i \leq n$ are $\theta$-stable irreducible components, and $\boldsymbol{d}_{i} \neq \boldsymbol{d}_{j}$ for all $1 \leq i \neq j \leq n$. Assume that

(1) $C$ contains the image of $X:=C_{1}^{m_{1}} \times \cdots \times C_{n}^{m_{n}}$ through the natural (diagonal) embedding $\mathscr{V}:=\bmod \left(Q, \boldsymbol{d}_{1}\right)^{m_{1}} \times \cdots \times \bmod \left(Q, \boldsymbol{d}_{n}\right)^{m_{n}} \hookrightarrow \bmod (Q, \boldsymbol{d}) ;$ and

(2) $C$ is a normal variety.

Then $\mathcal{M}(C)_{\theta}^{s s} \cong S^{m_{1}}\left(\mathcal{M}\left(C_{1}\right)_{\theta}^{s s}\right) \times \cdots \times S^{m_{n}}\left(\mathcal{M}\left(C_{n}\right)_{\theta}^{s s}\right)$.

Note that this reduction result allows us to "break" a moduli space of modules into smaller ones that are typically easier to handle; see Section 3C.

Recall that a quasitilted algebra is a basic and connected finite-dimensional algebra of the form End $\mathscr{H}(T)^{\mathrm{op}}$, where $\mathscr{H}$ is a hereditary category and $T \in \mathscr{H}$ is a tilting object. In [Happel et al. 1996, Theorem 2.3], Happel, Reiten, and Smalø proved that an algebra $A$ is quasitilted if and only if $A$ is of global dimension at most two and every indecomposable finite-dimensional $A$-module $X$ has projective dimension or injective dimension at most one. It was shown by Skowroński [1998, Theorem A] that a quasitilted algebra $A$ is tame if and only if its Tits form $q_{A}$ is weakly nonnegative.

Using our results described above, we can prove this:

Proposition 1.5. Let $A=k Q / I$ be a tame quasitilted algebra, $\boldsymbol{d}$ a dimension vector of $A$, and $C$ an irreducible component of $\bmod (A, \boldsymbol{d})$. 
(1) The field of rational invariants satisfies $k(C)^{\mathrm{GL}(d)} \simeq k\left(x_{1}, \ldots, x_{N}\right)$, where $N$ is the sum of the multiplicities of the isotropic imaginary roots that occur in the generic decomposition of $\boldsymbol{d}$ in $C$.

(2) If $\boldsymbol{d}$ is an isotropic root of $A$, then the moduli spaces $\mu(C)_{\theta}^{s s}$ for $\theta \in \mathbb{Z}^{Q_{0}}$ are products of projective spaces.

Our proof of Proposition 1.5(1) provides another approach to proving [Domokos and Lenzing 2002, Corollary 7.4].

The layout of this paper is as follows. In Section 2, we recall some background material on irreducible components of module varieties and their rational invariants. In Section 3, we first review King's construction of moduli spaces of modules for algebras, and then prove Theorem 1.3 in Section 3B. In Section 3C, we first explain how to extend Derksen and Weyman's notion [2011] of $\theta$-stable decomposition to quivers with relations, and then prove Theorem 1.4. We prove Theorem 1.1 and Proposition 1.5 in Section 4.

\section{Background on module varieties}

Let $Q=\left(Q_{0}, Q_{1}, t, h\right)$ be a finite quiver with vertex set $Q_{0}$ and arrow set $Q_{1}$. The two functions $t, h: Q_{1} \rightarrow Q_{0}$ assign to each arrow $a \in Q_{1}$ its tail $t a$ and head $h a$, respectively.

A representation $V$ of $Q$ over $k$ is a collection $(V(i), V(a))_{i \in Q_{0}, a \in Q_{1}}$ of finitedimensional $k$-vector spaces $V(i), i \in Q_{0}$, and $k$-linear maps

$$
V(a) \in \operatorname{Hom}_{k}(V(t a), V(h a)) \quad \text { for } a \in Q_{1} .
$$

The dimension vector of a representation $V$ of $Q$ is the function $\operatorname{dim} V: Q_{0} \rightarrow \mathbb{Z}$ defined by $(\operatorname{dim} V)(i)=\operatorname{dim}_{k} V(i)$ for $i \in Q_{0}$. Let $S_{i}$ be the one-dimensional representation of $Q$ at vertex $i \in Q_{0}$, and let us denote by $\boldsymbol{e}_{i}$ its dimension vector. By a dimension vector of $Q$, we simply mean a function $\boldsymbol{d} \in \mathbb{Z}_{\geq 0}^{Q_{0}}$.

Given two representations $V$ and $W$ of $Q$, we define a morphism $\varphi: V \rightarrow W$ to be a collection $(\varphi(i))_{i \in Q_{0}}$ of $k$-linear maps with $\varphi(i) \in \operatorname{Hom}_{k}(V(i), W(i))$ for each $i \in Q_{0}$, and such that $\varphi(h a) V(a)=W(a) \varphi(t a)$ for each $a \in Q_{1}$. We denote by $\operatorname{Hom}_{Q}(V, W)$ the $k$-vector space of all morphisms from $V$ to $W$. Let $V$ and $W$ be two representations of $Q$. We say that $V$ is a subrepresentation of $W$ if $V(i)$ is a subspace of $W(i)$ for each $i \in Q_{0}$ and $V(a)$ is the restriction of $W(a)$ to $V(t a)$ for each $a \in Q_{1}$. In this way, we obtain the abelian category $\operatorname{rep}(Q)$ of all representations of $Q$.

Given a quiver $Q$, its path algebra $k Q$ has a $k$-basis consisting of all paths (including the trivial ones), and the multiplication in $k Q$ is given by concatenation of paths. It is easy to see that any $k Q$-module defines a representation of $Q$, and vice-versa. Furthermore, the category $\bmod (k Q)$ of $k Q$-modules is equivalent to 
the category $\operatorname{rep}(Q)$. In what follows, we identify $\bmod (k Q)$ and $\operatorname{rep}(Q)$, and use the same notation for a module and the corresponding representation.

A two-sided ideal $I$ of $k Q$ is said to be admissible if there exists an integer $L \geq 2$ such that $R_{Q}^{L} \subseteq I \subseteq R_{Q}^{2}$. Here, $R_{Q}$ denotes the two-sided ideal of $k Q$ generated by all arrows of $Q$.

If $I$ is an admissible ideal of $K Q$, the pair $(Q, I)$ is called a bound quiver and the quotient algebra $k Q / I$ is called the bound quiver algebra of $(Q, I)$. Any admissible ideal is generated by finitely many admissible relations, and any bound quiver algebra is finite-dimensional and basic. Moreover, a bound quiver algebra $k Q / I$ is connected if and only if (the underlying graph of) $Q$ is connected; see for example [Assem et al. 2006].

It is well known that any basic algebra $A$ is isomorphic to the bound quiver algebra of a bound quiver $\left(Q_{A}, I\right)$, where $Q_{A}$ is the Gabriel quiver of $A$; see [Assem et al. 2006]. (Note that the ideal of relations $I$ is not uniquely determined by $A$.) We say that $A$ is a triangular algebra if its Gabriel quiver has no oriented cycles.

Fix a bound quiver $(Q, I)$ and let $A=k Q / I$ be its bound quiver algebra. We denote by $e_{i}$ the primitive idempotent corresponding to the vertex $i \in Q_{0}$. A representation $M$ of a $A$ (or $(Q, I))$ is just a representation $M$ of $Q$ such that $M(r)=0$ for all $r \in I$. The category $\bmod (A)$ of finite-dimensional left $A$-modules is equivalent to the category $\operatorname{rep}(A)$ of representations of $A$. As before, we identify $\bmod (A)$ and $\operatorname{rep}(A)$, and make no distinction between $A$-modules and representations of $A$.

Assume from now on that $A$ has finite global dimension; this happens, for example, when $Q$ has no oriented cycles. The Ringel form of $A$ is the bilinear form $\langle\cdot, \cdot\rangle_{A}: \mathbb{Z}^{Q_{0}} \times \mathbb{Z}^{Q_{0}} \rightarrow \mathbb{Z}$ defined by

$$
\langle\boldsymbol{d}, \boldsymbol{e}\rangle_{A}=\sum_{l \geq 0}(-1)^{l} \sum_{i, j \in Q_{0}} \operatorname{dim}_{k} \operatorname{Ext}_{A}^{l}\left(S_{i}, S_{j}\right) \boldsymbol{d}(i) \boldsymbol{e}(j) .
$$

Note that if $M$ is a $\boldsymbol{d}$-dimensional $A$-module and $N$ is an $\boldsymbol{e}$-dimensional $A$-module, then

$$
\langle\boldsymbol{d}, \boldsymbol{e}\rangle_{A}=\sum_{l \geq 0}(-1)^{l} \operatorname{dim}_{k} \operatorname{Ext}_{A}^{l}(M, N)
$$

The quadratic form induced by $\langle\cdot, \cdot\rangle_{A}$ is denoted by $\chi_{A}$.

The Tits form of $A$ is the integral quadratic form $q_{A}: \mathbb{Z}^{Q_{0}} \rightarrow \mathbb{Z}$ defined by

$$
\begin{aligned}
q_{A}(\boldsymbol{d}):=\sum_{i \in Q_{0}} \boldsymbol{d}^{2}(i)-\sum_{i, j \in Q_{0}} \operatorname{dim}_{k} \operatorname{Ext}_{A}^{1}\left(S_{i}, S_{j}\right) \boldsymbol{d}(i) \boldsymbol{d}(j) & \\
& +\sum_{i, j \in Q_{0}} \operatorname{dim}_{k} \operatorname{Ext}_{A}^{2}\left(S_{i}, S_{j}\right) \boldsymbol{d}(i) \boldsymbol{d}(j) .
\end{aligned}
$$


If $A$ is triangular, then $r(i, j):=\left|R \cap e_{j}\langle R\rangle e_{i}\right|$ is precisely $\operatorname{dim}_{k} \operatorname{Ext}_{A}^{2}\left(S_{i}, S_{j}\right)$, for all $i, j \in Q_{0}$, as shown by Bongartz [1983]. So, in the triangular case, we can write

$$
q_{A}(\boldsymbol{d})=\sum_{i \in Q_{0}} \boldsymbol{d}^{2}(i)-\sum_{a \in Q_{1}} \boldsymbol{d}(t a) \boldsymbol{d}(h a)+\sum_{i, j \in Q_{0}} r(i, j) \boldsymbol{d}(i) \boldsymbol{d}(j) .
$$

2A. The generic decomposition for irreducible components. Let $\boldsymbol{d}$ be a dimension vector of $A$ (or equivalently, of $Q$ ). The variety of $\boldsymbol{d}$-dimensional $A$-modules is the affine variety

$$
\bmod (A, \boldsymbol{d})=\left\{M \in \prod_{a \in Q_{1}} \operatorname{Mat}_{\boldsymbol{d}(h a) \times \boldsymbol{d}(t a)}(k) \mid M(r)=0 \text { for all } r \in I\right\} .
$$

It is clear that $\bmod (A, \boldsymbol{d})$ is a $\operatorname{GL}(\boldsymbol{d})$-invariant closed subset of the affine space $\bmod (Q, \boldsymbol{d}):=\prod_{a \in Q_{1}} \operatorname{Mat}_{\boldsymbol{d}(h a) \times \boldsymbol{d}(t a)}(k)$. Note that $\bmod (A, \boldsymbol{d})$ does not have to be irreducible. We call $\bmod (A, \boldsymbol{d})$ the module variety of $\boldsymbol{d}$-dimensional $A$-modules. We also denote by $\operatorname{ind}(A, \boldsymbol{d})$ the (possibly empty) constructible subset of all indecomposable modules in $\bmod (A, \boldsymbol{d})$.

Let $C$ be an irreducible component of $\bmod (A, \boldsymbol{d})$. We say that $C$ is indecomposable if $C$ has a nonempty open subset of indecomposable modules. We call $C$ a $S c h u r$ irreducible component if $C$ contains a Schur $A$-module. (Recall that a Schur $A$-module is just an $A$-module $M$ such that $\operatorname{End}_{A}(M) \simeq k$.) Note that a Schur irreducible component is always indecomposable. The converse is always true for path algebras of quivers without oriented cycles. Finally, we say that $\boldsymbol{d}$ is a generic root of $A$ if $\bmod (A, \boldsymbol{d})$ has an indecomposable irreducible component.

Let us consider a decomposition $\boldsymbol{d}=\boldsymbol{d}_{1}+\cdots+\boldsymbol{d}_{t}$, where $\boldsymbol{d}_{i} \in \mathbb{Z}_{\geq 0}^{Q_{0}}$ for $1 \leq i \leq t$. If $C_{i}$ is a $\operatorname{GL}\left(\boldsymbol{d}_{i}\right)$-invariant subset of $\bmod \left(A, \boldsymbol{d}_{i}\right)$ for $1 \leq i \leq t$, we denote by $C_{1} \oplus \cdots \oplus C_{t}$ the constructible subset of $\bmod (A, \boldsymbol{d})$ consisting of all modules isomorphic to direct sums of the form $\bigoplus_{i=1}^{t} X_{i}$ with $X_{i} \in C_{i}$ for all $1 \leq i \leq t$.

As shown by de la Peña [1991, Section 1.3] and Crawley-Boevey and Schröer [2002, Theorem 1.1], if $C$ is an irreducible component of $\bmod (A, \boldsymbol{d})$, then there are unique generic roots $\boldsymbol{d}_{1}, \ldots, \boldsymbol{d}_{t}$ of $A$ such that $\boldsymbol{d}=\boldsymbol{d}_{1}+\cdots+\boldsymbol{d}_{t}$ and

$$
C=\overline{C_{1} \oplus \cdots \oplus C_{t}}
$$

for some indecomposable irreducible components $C_{i}$ of $\bmod \left(A, \boldsymbol{d}_{i}\right)$ for $1 \leq i \leq t$. Also, the indecomposable irreducible components $C_{i}$ for $1 \leq i \leq t$ are uniquely determined by this property. We call $\boldsymbol{d}=\boldsymbol{d}_{1} \oplus \cdots \oplus \boldsymbol{d}_{t}$ the generic decomposition of $\boldsymbol{d}$ in $C$, and $C=\overline{C_{1} \oplus \cdots \oplus C_{t}}$ the generic decomposition of $C$.

Recall that for an irreducible component $C \subseteq \bmod (A, \boldsymbol{d})$, the field of rational $\mathrm{GL}(\boldsymbol{d})$-invariants on $C$ is

$$
k(C)^{\mathrm{GL}(\boldsymbol{d})}=\{\phi \in k(C) \mid g \cdot \phi=\phi \text { for all } g \in \mathrm{GL}(\boldsymbol{d})\} .
$$


In what follows, if $R$ is an integral domain, we denote its field of fractions by $\operatorname{Quot}(R)$. Moreover, if $K / k$ is a field extension and $m$ is a positive integer, we define $S^{m}(K / k)$ to be the field $\left(\operatorname{Quot}\left(K^{\otimes m}\right)\right)^{S_{m}}$, which is in fact the same as $\operatorname{Quot}\left(\left(K^{\otimes m}\right)^{S_{m}}\right)$, since $S_{m}$ is a finite group.

Proposition 2.1 [Chindris 2011, Proposition 4.7]. Assume that the generic decomposition of $C$ is of the form

$$
C=\overline{C_{1}^{\oplus m_{1}} \oplus \cdots \oplus C_{n}^{\oplus m_{n}}}
$$

where $C_{i} \subseteq \bmod \left(A, \boldsymbol{d}_{i}\right)$ for $1 \leq i \leq n$ are indecomposable irreducible components, $m_{1}, \ldots, m_{n}$ are positive integers, and $\boldsymbol{d}_{i} \neq \boldsymbol{d}_{j}$ for all $1 \leq i \neq j \leq n$. Then

$$
k(C)^{\mathrm{GL}(\boldsymbol{d})} \simeq \operatorname{Quot}\left(\bigotimes_{i=1}^{n} S^{m_{i}}\left(k\left(C_{i}\right)^{\mathrm{GL}\left(\boldsymbol{d}_{i}\right)} / k\right)\right) .
$$

In the next section, we present a homological method for studying fields of rational invariants on indecomposable irreducible components in module varieties.

2B. Exceptional sequences and rational invariants. Recall that a sequence $\mathscr{E}=$ $\left(E_{1}, \ldots, E_{t}\right)$ of $A$-modules is called an orthogonal exceptional sequence if the following conditions are satisfied:

(1) $E_{i}$ is an exceptional $A$-module, that is, $\operatorname{End}_{A}\left(E_{i}\right)=k$ and $\operatorname{Ext}_{A}^{l}\left(E_{i}, E_{i}\right)=0$ for all $l \geq 1$ and $1 \leq i \leq t$.

(2) $\operatorname{Ext}_{A}^{l}\left(E_{i}, E_{j}\right)=0$ for all $l \geq 0$ and $1 \leq i<j \leq t$.

(3) $\operatorname{Hom}_{A}\left(E_{j}, E_{i}\right)=0$ for all $1 \leq i<j \leq t$.

Given an orthogonal exceptional sequence $\mathscr{E}$, consider the full subcategory filt: of $\bmod (A)$ whose objects $M$ have a finite filtration $0=M_{0} \subseteq M_{1} \subseteq \cdots \subseteq M_{s}=M$ of submodules such that each factor $M_{j} / M_{j-1}$ is isomorphic to one of the $E_{1}, \ldots, E_{t}$. For a dimension vector $\boldsymbol{d}$ of $A$, we define

$$
\text { filt } \mathscr{E}_{\mathscr{C}}(\boldsymbol{d})=\{M \in \bmod (A, \boldsymbol{d}) \mid M \text { is isomorphic to a module in filt } \mathscr{\mathscr { E }}\} .
$$

We will be especially interested in short orthogonal exceptional sequences. As a first step in proving the rationality of fields of rational invariants for $A$, we will use the following direct consequence of the reduction theorem [Chindris 2011, Theorem 1.2]:

Proposition 2.2. Let $\boldsymbol{d}$ be a generic root of $A$ and let $C \subseteq \bmod (A, \boldsymbol{d})$ be an indecomposable irreducible component. Assume that there exists an orthogonal exceptional sequence $\mathscr{E}=\left(E_{1}, E_{2}\right)$ of $A$-modules such that $d=\operatorname{dim} E_{1}+\operatorname{dim} E_{2}$, filte $\mathscr{E}(\boldsymbol{d}) \cap C \neq \varnothing$, and $\operatorname{dim}_{\operatorname{Ext}_{A}^{2}}\left(E_{2}, E_{1}\right)=0$. Then $k(C) \mathrm{GL}(\boldsymbol{d}) \simeq k\left(x_{1}, \ldots, x_{n-1}\right)$ where $n=\operatorname{dim}_{k} \operatorname{Ext}_{A}^{1}\left(E_{2}, E_{1}\right)$. 
Proof. The triangular algebra $A_{\mathscr{E}}$ that arises from the (minimal) $A_{\infty}$-algebra structure of the Yoneda algebra $\operatorname{Ext}_{A}^{\circ}\left(E_{1} \oplus E_{2}, E_{1} \oplus E_{2}\right)$ is precisely the path algebra of the generalized Kronecker quiver, $K_{n}$, with two vertices and $n$ arrows, all pointing in the same direction. It now follows from [Chindris 2011, Theorem 1.2] that $k(C)^{\mathrm{GL}(\boldsymbol{d})} \simeq k\left(\bmod \left(K_{n},(1,1)\right)\right)^{\mathrm{GL}((1,1))} \simeq k\left(x_{1}, x_{2}, \ldots x_{n-1}\right)$.

\section{Moduli spaces of modules}

Let $A=k Q / I$ be a bound quiver algebra and let $\boldsymbol{d} \in \mathbb{Z}_{\geq 0}^{Q_{0}}$ be a dimension vector of $A$. We denote $\operatorname{GL}(\boldsymbol{d}) / T_{1}$ by PGL $(\boldsymbol{d})$, where $T_{1}=\left\{\left(\lambda \operatorname{Id}_{\boldsymbol{d}(i)}\right)_{i \in Q_{0}} \mid \lambda \in k^{*}\right\} \leq \mathrm{GL}(\boldsymbol{d})$. Note that there is a well-defined action of $\operatorname{PGL}(\boldsymbol{d})$ on $\bmod (A, \boldsymbol{d})$ since $T_{1}$ acts trivially on $\bmod (A, \boldsymbol{d})$.

We always identify $K_{0}(A)$ with the lattice $\mathbb{Z}^{Q_{0}}$, which, in turn, we identify with $\operatorname{Hom}_{\mathbb{Z}}\left(K_{0}(A), \mathbb{Z}\right)$ via $\theta(\boldsymbol{d})=\sum_{i \in Q_{0}} \theta(i) \boldsymbol{d}(i)$ for all $\theta \in \mathbb{Z}^{Q_{0}}$ and $\boldsymbol{d} \in \mathbb{Z}^{Q_{0}}$. Note that when $A$ is triangular, any integral weight $\theta \in \mathbb{Z}^{Q_{0}}$ can be written as $\langle\boldsymbol{d}, \cdot\rangle_{A}$ for a unique vector $\boldsymbol{d} \in \mathbb{Z}^{Q_{0}}$. Similarly, $\theta$ can be written as $\langle\cdot, \boldsymbol{e}\rangle_{A}$ for a unique vector $\boldsymbol{e} \in \mathbb{Z}^{Q_{0}}$.

Note that any $\theta \in \mathbb{Z}^{Q_{0}}$ defines a rational character $\chi_{\theta}: \operatorname{GL}(\boldsymbol{d}) \rightarrow k^{*}$ by

$$
\chi_{\theta}\left((g(i))_{i \in Q_{0}}\right)=\prod_{i \in Q_{0}}(\operatorname{det} g(i))^{\theta(i)} .
$$

In this way, we can identify $\mathbb{Z}^{Q_{0}}$ with the group $X^{\star}(\mathrm{GL}(\boldsymbol{d}))$ of rational characters of $\operatorname{GL}(\boldsymbol{d})$, assuming that $\boldsymbol{d}$ is a sincere dimension vector. In general, we have only the natural epimorphism $\mathbb{Z}^{Q_{0}} \rightarrow X^{*}(\mathrm{GL}(\boldsymbol{d}))$.

Now, let $\theta \in \mathbb{Z}^{Q_{0}}$ be an integral weight of $A$. Following King [1994], an $A$-module $M$ is said to be $\theta$-semistable if $\theta(\operatorname{dim} M)=0$ and $\theta\left(\operatorname{dim} M^{\prime}\right) \leq 0$ for all submodules $M^{\prime} \leq M$. We say that $M$ is $\theta$-stable if $M$ is nonzero, $\theta(\operatorname{dim} M)=0$, and $\theta\left(\operatorname{dim} M^{\prime}\right)<0$ for all submodules $\{0\} \neq M^{\prime}<M$. Now, consider the (possibly empty) open subsets

$$
\bmod (A, \boldsymbol{d})_{\theta}^{S S}=\{M \in \bmod (A, \boldsymbol{d}) \mid M \text { is } \theta \text {-semistable }\}
$$

and

$$
\bmod (A, \boldsymbol{d})_{\theta}^{s}=\{M \in \bmod (A, \boldsymbol{d}) \mid M \text { is } \theta \text {-stable }\}
$$

of $\boldsymbol{d}$-dimensional $\theta$ (-semi)-stable $A$-modules.

The weight space of semiinvariants on $\bmod (A, \boldsymbol{d})$ of weight $n \theta \in \mathbb{Z}^{Q_{0}}$, where $n \in \mathbb{Z}_{\geq 0}$, is

$$
\operatorname{SI}(A, \boldsymbol{d})_{n \theta}:=\{f \in k[\bmod (A, \boldsymbol{d})] \mid g \cdot f=(n \theta)(g) f \text { for all } g \in \mathrm{GL}(\boldsymbol{d})\} .
$$


Using methods from GIT, King [1994] showed that the projective variety

$$
M(A, \boldsymbol{d})_{\theta}^{s s}:=\operatorname{Proj}\left(\bigoplus_{n \geq 0} \operatorname{SI}(A, \boldsymbol{d})_{n \theta}\right)
$$

is a GIT-quotient of $\bmod (A, \boldsymbol{d})_{\theta}^{s s}$ by the action of $\operatorname{PGL}(\boldsymbol{d})$. We say that $\boldsymbol{d}$ is a $\theta$-semistable dimension vector if $\bmod (A, \boldsymbol{d})_{\theta}^{s s} \neq \varnothing$.

For an irreducible component $C \subseteq \bmod (A, \boldsymbol{d})$, we similarly define $C_{\theta}^{s s}, C_{\theta}^{s}$, $\operatorname{SI}(C)_{n \theta}$, and $M(C)_{\theta}^{s S}$.

3A. Families of A-modules. Let us denote by $\bmod (A)_{\theta}^{S s}$ the full subcategory of $\bmod (A)$ consisting of the $\theta$-semistable modules. It is easy to see that $\bmod (A)_{\theta}^{s s}$ is a full exact abelian subcategory of $\bmod (A)$ that is closed under extensions and whose simple objects are precisely the $\theta$-stable modules. Moreover, $\bmod (A)_{\theta}^{S S}$ is Artinian and Noetherian, and hence every $\theta$-semistable $A$-module has a JordanHölder filtration in $\bmod (A)_{\theta}^{s s}$.

Two $\theta$-semistable $A$-modules are said to be $S$-equivalent if they have the same composition factors in $\bmod (A)_{\theta}^{s S}$. It was proved in [King 1994, Proposition 4.2] that the points of $M(A, \boldsymbol{d})_{\theta}^{s s}$ are in one-to-one correspondence with the $S$-equivalence classes of $\boldsymbol{d}$-dimensional $\theta$-semistable $A$-modules.

We now recall the definition of a family of $A$-modules over a variety that was introduced in this context by King [1994]. Let $Z$ be a (reduced) variety and let $\left(V_{z}\right)_{z \in Z}$ be a collection of $A$-modules parametrized by $Z$. Following the presentation in [Domokos and Lenzing 2000, Section 6], we call $\left(V_{z}\right)_{z \in Z}$ a family of A-modules if the following two conditions are satisfied:

(i) $\left(V_{z}\right)_{z \in Z}$ is an algebraic vector bundle over $Z$; in particular, the vector spaces $V_{z}$ for $z \in Z$ have the same dimension.

(ii) For each $a \in A$, the map $z \rightarrow a \cdot \operatorname{Id}_{V_{z}}(z \in Z)$ is a section of the endomorphism bundle $\left(\operatorname{End}_{k}\left(V_{z}\right)\right)_{z \in Z}$; in other words, the $A$-module structure on $V_{z}$ varies algebraically with $z \in Z$.

King showed that $\mathcal{M}(A, \boldsymbol{d})_{\theta}^{s S}$ is a coarse moduli space for families of $\boldsymbol{d}$-dimensional $\theta$-semistable $A$-modules; see [King 1994, Proposition 5.2]. This essentially says that if $\left(V_{z}\right)_{z \in Z}$ is a family of $\boldsymbol{d}$-dimensional $\theta$-semistable $A$-modules and $\phi$ is the (unique) set-theoretic map $Z \rightarrow M(A, \boldsymbol{d})_{\theta}^{s s}$ that sends each $z \in Z$ to the point representing the $S$-equivalence class of $V_{z}$, then $\phi$ is a morphism of varieties.

Lemma 3.1. Let $A$ and $B$ be two bound quiver algebras, $T$ an $A$-B-bimodule, $Z$ a variety, and $n$ a positive integer.

(1) Let $\left(V_{z}\right)_{z \in Z}$ be a family of A-modules parametrized by $Z$. Assume that for each $0 \leq l \leq n$, there exists an integer $m_{l}$ such that $\operatorname{dim}_{k} \operatorname{Ext}_{A}^{l}\left(T, V_{z}\right)=m_{l}$ for all $z \in Z$. Then $\left(\operatorname{Ext}_{A}^{n}\left(T, V_{z}\right)\right)_{z \in Z}$ is a family of $B$-modules. 
(2) Let $\left(W_{z}\right)_{z \in Z}$ be a family of B-modules parametrized by $Z$. Assume that for each $0 \leq l \leq n$, there exists an integer $t_{l}$ such that $\operatorname{dim}_{k} \operatorname{Tor}_{B}^{l}\left(T, W_{z}\right)=t_{l}$ for all $z \in Z$. Then $\left(\operatorname{Tor}_{B}^{n}\left(T, W_{z}\right)\right)_{z \in Z}$ is a family of $A$-modules.

Remark 1. For $n=1$, this lemma was proved by Domokos and Lenzing [2000, Lemma 6.3]. Here, we explain how to prove the general case by working with Hochschild complexes.

Proof. In what follows, for a given integer $l \geq 0$, we write $A^{l}$ and $B^{l}$ for

$$
\underbrace{A \otimes_{k} \cdots \otimes_{k} A}_{l} \text { and } \underbrace{B \otimes_{k} \cdots \otimes_{k} B}_{l}
$$

(1) For each $z \in Z$, we consider the Hochschild complex

$K_{z}^{*}: 0 \rightarrow \operatorname{Hom}_{k}\left(T, V_{z}\right) \stackrel{d_{z}^{0}}{\longrightarrow} \operatorname{Hom}_{k}\left(A \otimes_{k} T, V_{z}\right) \stackrel{d_{z}^{1}}{\longrightarrow} \operatorname{Hom}_{k}\left(A^{2} \otimes_{k} T, V_{z}\right) \longrightarrow \cdots$, where

$$
\begin{aligned}
& d_{z}^{l}\left(\phi_{l}\right)\left(a_{1} \otimes \cdots \otimes a_{l+1} \otimes t\right) \\
& \quad=a_{1} \phi_{l}\left(a_{2} \otimes \cdots \otimes a_{l+1} \otimes t\right)+\sum_{i=1}^{l}(-1)^{i} \phi_{l}\left(a_{1} \otimes \cdots \otimes\left(a_{i} a_{i+1}\right) \otimes \cdots \otimes t\right) \\
& \quad+(-1)^{l+1} \phi_{l}\left(a_{1} \otimes \cdots \otimes a_{l} \otimes\left(a_{l+1} t\right)\right) .
\end{aligned}
$$

As $k$ is a commutative field, we know that $H^{l}\left(K_{z}^{*}\right) \simeq \operatorname{Ext}_{A}^{l}\left(T, V_{z}\right)$ for all $l \geq 0$; see, for example, [Weibel 1994, Theorem 8.7.10 and Lemma 9.1.9].

It is now easy to see that $\left(d_{z}^{l}\right)_{z \in Z}$ is a morphism of vector bundles for each integer $l \geq 0$. Also, for each $0 \leq l \leq n$, the maps $d_{z}^{l}$ for $z \in Z$, have constant rank, and hence the kernel and the image of $\left(d_{z}^{l}\right)_{z \in Z}$ are subbundles of $\left(\operatorname{Hom}_{k}\left(A^{l} \otimes_{k} T, V_{z}\right)\right)_{z \in Z}$ and $\left(\operatorname{Hom}_{k}\left(A^{l+1} \otimes_{k} T, V_{z}\right)\right)_{z \in Z}$, respectively [Le Potier 1997, Proposition 1.7.2]. Since these subbundles are clearly families of $B$-modules, $\left(\operatorname{Ext}_{A}^{n}\left(T, V_{z}\right)\right)_{z \in Z}$ is indeed a family of $B$-modules.

(2) For this part, we work with the homology of the following complex (see for example [Weibel 1994, Section 8.7.1]):

$$
K_{z}^{*}: 0 \longleftarrow T \otimes_{k} W_{z} \stackrel{\left(d_{0}\right)_{z}}{\longleftarrow} T \otimes_{k} B \otimes_{k} W_{z} \stackrel{\left(d_{1}\right)_{z}}{\longleftarrow} T \otimes_{k} B^{2} \otimes_{k} W_{z} \longleftarrow \cdots .
$$

As before, the differentials of this complex give rise to morphisms of vector bundles whose kernels and images are families of $A$-modules. From this, one immediately derives the desired claim. 
3B. Moduli spaces and tilting. We now explain how moduli spaces of semistable $A$-modules behave under tilting. This was already discussed by Domokos and Lenzing [2000] in the context of moduli spaces of modules over canonical algebras.

Let $T$ be a basic tilting $A$-module and denote $\operatorname{End}_{A}(T)^{\text {op }}$ by $B$. The torsion pairs $(\mathscr{T}(T), \mathscr{F}(T))$ in $\bmod (A)$ induced by $T$ and $(\mathscr{X}(T), \mathscr{Y}(T))$ in $\bmod (B)$ induced by $D(T):=\operatorname{Hom}_{k}(T, k)$ are

- $\mathscr{T}\left({ }_{A} T\right)=\left\{M \in \bmod (A) \mid \operatorname{Ext}_{A}^{1}(T, M)=0\right\} ;$

- $\mathscr{F}\left({ }_{A} T\right)=\left\{M \in \bmod (A) \mid \operatorname{Hom}_{A}(T, M)=0\right\}$;

- $\mathscr{X}\left(T_{B}\right)=\left\{N \in \bmod (B) \mid \operatorname{Hom}_{B}(N, D(T))=0\right\}$

$$
=\left\{N \in \bmod (B) \mid T \otimes_{B} N=0\right\} ; \text { and }
$$

- $y\left(T_{B}\right)=\left\{N \in \bmod (B) \mid \operatorname{Ext}_{B}^{1}(N, D(T))=0\right\}$.

$$
=\left\{N \in \bmod (B) \mid \operatorname{Tor}_{B}^{1}(T, N)=0\right\}
$$

The Brenner-Butler tilting theorem (see for example [Assem et al. 2006]) tells us that the tilting functor $\operatorname{Hom}_{A}\left(T,_{-}\right): \bmod (A) \rightarrow \bmod (B)$ induces an equivalence of categories between $\mathscr{T}(T)$ and $\mathcal{Y}(T)$ with quasiinverse $T \otimes_{B_{-}}$. Furthermore, the functor $\operatorname{Ext}_{A}^{1}\left(T,,_{-}\right): \bmod (B) \rightarrow \bmod (A)$ induces an equivalence of categories between $\mathscr{F}(T)$ and $\mathscr{L}(T)$ with quasiinverse $\operatorname{Tor}_{1}^{B}\left(T,_{-}\right)$.

We also have the isometry $u: K_{0}(A) \rightarrow K_{0}(B)$ defined by

$$
u(\operatorname{dim} M)=\operatorname{dim} \operatorname{Hom}_{A}(T, M)-\operatorname{dim} \operatorname{Ext}_{A}^{1}(T, M)
$$

for any $A$-module $M$.

Definition 3.2. We say an integral weight $\theta \in \operatorname{Hom}_{\mathbb{Z}}\left(K_{0}(A), \mathbb{Z}\right)$ is well-positioned with respect to $T$ if either

(1) there are nonzero $\theta$-semistable $A$-modules, $\bmod (A)_{\theta}^{s s} \subseteq \mathscr{T}(T)$, and

$$
\theta(\operatorname{dim} M)<0
$$

for all nonzero modules $M$ in $\mathscr{F}(T)$; or

(2) there are nonzero $\theta$-semistable $A$-modules, $\bmod (A)_{\theta}^{s s} \subseteq \mathscr{F}(T)$, and

$$
\theta(\operatorname{dim} M)>0
$$

for all nonzero modules $M$ in $\mathscr{T}(T)$.

Let $\theta$ be an integral weight of $A$ that is well positioned with respect to $T$. We define $\left|\theta \circ u^{-1}\right|$ to be $\theta \circ u^{-1}$ if condition (1) above is satisfied; if condition (2) is satisfied, $\left|\theta \circ u^{-1}\right|$ is defined to be $-\theta \circ u^{-1}$.

Now we are ready to prove Theorem 1.3: 
Proof of Theorem 1.3. (a) Case 1: $\bmod (A)_{\theta}^{s s} \subseteq \mathcal{T}(T)$ and $\theta(\operatorname{dim} M)<0$ for all nonzero modules $M$ in $\mathscr{F}(T)$. In this case, $\theta^{\prime}=\theta \circ u^{-1}$ and $F=\operatorname{Hom}_{A}\left(T,_{-}\right)$.

Let $M$ be a $\theta$-semistable $A$-module. We show that $N=F(M)$ is $\theta^{\prime}$-semistable. As $M$ is a $\theta$-semistable module lying in $\mathscr{T}(T)$, we deduce that $\theta^{\prime}(\operatorname{dim} N)=0$. Now, let $N^{\prime}$ be a submodule of $N$ and let $M^{\prime} \in \mathscr{T}(T)$ be such that $F\left(M^{\prime}\right) \simeq N^{\prime}$. In particular, we get that $\theta^{\prime}\left(\operatorname{dim} N^{\prime}\right)=\theta^{\prime}\left(u\left(\operatorname{dim} M^{\prime}\right)=\theta\left(\operatorname{dim} M^{\prime}\right)\right.$. If $\phi \in \operatorname{Hom}_{A}\left(M^{\prime}, M\right)$ is the morphism corresponding to the inclusion $N^{\prime} \hookrightarrow N$, then $\operatorname{ker}(\phi) \in \mathscr{F}(T)$ as $F$ is left exact. Using our assumption on $\theta$, it is now clear that $\theta^{\prime}\left(\operatorname{dim} N^{\prime}\right) \leq 0$. This shows that $N$ is $\theta^{\prime}$-semistable.

Now, let $\widetilde{N}$ be a $\theta^{\prime}$-semistable $B$-module. First, we claim that $\widetilde{N} \in \mathcal{Y}(T)$. Indeed, let us consider the canonical sequence of $\widetilde{N}$ with respect to $(\mathscr{L}(T)$, $\mathscr{Y}(T))$ :

$$
0 \rightarrow \operatorname{Ext}_{A}^{1}\left(T, \operatorname{Tor}_{B}^{1}(T, \tilde{N})\right) \longrightarrow \tilde{N} \longrightarrow \operatorname{Hom}_{A}\left(T, T \otimes_{B} \tilde{N}\right) \rightarrow 0 .
$$

If $\widetilde{N}^{\prime}$ denotes the $B$-module $\operatorname{Ext}_{A}^{1}\left(T, \operatorname{Tor}_{B}^{1}(T, \tilde{N})\right)$, then

$$
\operatorname{dim} \tilde{N}^{\prime}=-u\left(\operatorname{dim} \operatorname{Tor}_{B}^{1}(T, \tilde{N})\right),
$$

and so $\theta^{\prime}\left(\operatorname{dim} \tilde{N}^{\prime}\right)=-\theta\left(\operatorname{dim} \operatorname{Tor}_{B}^{1}(T, \tilde{N})\right)$. Using again our assumption on $\theta$, we have that $\theta^{\prime}\left(\operatorname{dim} \widetilde{N}^{\prime}\right)$ is strictly positive unless $\operatorname{Tor}_{B}^{1}(T, \widetilde{N})=\{0\}$. But since $\widetilde{N}$ is $\theta^{\prime}$-semistable, we must have $\operatorname{Tor}_{B}^{1}(T, \widetilde{N})=\{0\}$, and hence $\widetilde{N} \simeq F(\tilde{M})$, where $\widetilde{M}:=T \otimes_{B} \widetilde{N} \in \mathscr{T}(T)$.

Next, we show that $\tilde{M}$ is $\theta$-semistable. It is clear that $\theta(\operatorname{dim} \tilde{M})=0$. Now, let $\widetilde{M}^{\prime}$ be a submodule of $\widetilde{M}$ and note that coker $F(\pi) \in \mathscr{X}(T)$, where $\pi: \widetilde{M} \rightarrow$ $\widetilde{M} / \tilde{M}^{\prime}$ is the canonical projection. So, there exists an $A$-module $\widetilde{M}^{\prime \prime}$ in $\mathscr{F}(T)$ such that $\operatorname{dim} \operatorname{coker}(F(\pi))=\operatorname{dim} \operatorname{Ext}_{A}^{1}\left(T, \tilde{M}^{\prime \prime}\right)=-u\left(\operatorname{dim} \tilde{M}^{\prime \prime}\right)$. In particular, we get that $\theta^{\prime}(\operatorname{dim} \operatorname{coker} F(\pi))=-\theta\left(\operatorname{dim} \tilde{M}^{\prime \prime}\right) \geq 0$, and from this we see that $\theta^{\prime}\left(\operatorname{dim} F\left(\tilde{M} / \tilde{M}^{\prime}\right)\right) \geq 0$. But since $\theta^{\prime}\left(\operatorname{dim} F\left(\tilde{M} / \tilde{M}^{\prime}\right)\right)=\theta\left(\operatorname{dim} \tilde{M} / \tilde{M}^{\prime}\right)$, we conclude that $\theta\left(\operatorname{dim} \tilde{M}^{\prime}\right) \leq 0$. This proves part (a) in Case 1 .

Case 2: $\bmod (A)_{\theta}^{s S} \subseteq \mathscr{F}(T)$ and $\theta(\operatorname{dim} M)>0$ for all nonzero modules $M$ in $\mathscr{T}(T)$. In this case, $\theta^{\prime}=-\theta \circ u^{-1}$ and $F=\operatorname{Ext}_{A}^{1}\left(T,_{-}\right)$. The proof in this case is essentially dual to the one above; one simply uses the existence of long exact sequences in (co)homology along with the fact that the projective dimension of $T$ is at most one.

(b) For this part, we follow closely the arguments in [Domokos and Lenzing 2000, Section 6]. First, let us consider the canonical family $\left(V_{M}\right)_{M \in \bmod (A, \boldsymbol{d})_{\theta}^{s s}}$ of $\boldsymbol{d}$ dimensional $\theta$-semistable $A$-modules. By this we simply mean the trivial vector bundle $\bmod (A, \boldsymbol{d})_{\theta}^{s S} \times V$, where $V=\bigoplus_{i \in Q_{0}} k^{\boldsymbol{d}(i)}$ and, for each $M \in \bmod (A, \boldsymbol{d})_{\theta}^{s s}$, $V$ is equipped with the $A$-module structure corresponding to $M$. Now, it follows from part (a) that for each $M \in \bmod (A, \boldsymbol{d})_{\theta}^{s s}, \quad F\left(V_{M}\right)$ is a $\boldsymbol{d}^{\prime}$-dimensional $\theta^{\prime}$-semistable $B$-module. Consequently, we can apply Lemma 3.1 to conclude 
that $\left(F\left(V_{M}\right)\right)_{M \in \bmod (A, \boldsymbol{d})_{\theta}^{s s}}$ is actually a family of $\boldsymbol{d}^{\prime}$-dimensional $\theta^{\prime}$-semistable $B$ modules. Hence, we get the morphism of varieties $\phi: \bmod (A, \boldsymbol{d})_{\theta}^{s s} \rightarrow \mathcal{M}\left(B, \boldsymbol{d}^{\prime}\right)_{\theta^{\prime}}^{s s}$ that sends $M \in \bmod (A, \boldsymbol{d})_{\theta}^{s s}$ to the point of $M\left(B, \boldsymbol{d}^{\prime}\right)_{\theta^{\prime}}^{s s}$ corresponding to the $S$ equivalence class of $F\left(V_{M}\right)$. It is clear that $\phi$ is a $\operatorname{PGL}(\boldsymbol{d})$-invariant morphism. From the universal property of the GIT-quotient $\mathcal{M}(A, \boldsymbol{d})_{\theta}^{s s}$, we obtain the morphism of algebraic varieties $f: \mathcal{M}(A, \boldsymbol{d})_{\theta}^{s s} \rightarrow \mathcal{M}\left(B, \boldsymbol{d}^{\prime}\right)_{\theta^{\prime}}$ for which $f \circ \pi=\phi$, where $\pi: \bmod (A, \boldsymbol{d})_{\theta}^{s s} \rightarrow \mathcal{M}(A, \boldsymbol{d})_{\theta}^{s s}$ is the quotient morphism. To construct the inverse morphism of $f$, one follows the same arguments as above, with the functor $F$ replaced by its quasiinverse.

3C. The theta-stable decomposition for irreducible components. Derksen and Weyman [2011] introduced the notion of $\theta$-stable decomposition for spaces of representations of quivers without relations. In this section, we explain how to extend [Derksen and Weyman 2011, Theorem 3.20] to quivers with relations.

Let $A=k Q / I$ be a bound quiver algebra, $\boldsymbol{d} \in \mathbb{Z}_{\geq 0}^{Q_{0}}$ a dimension vector of $A$, $C \subseteq \bmod (A, \boldsymbol{d})$ an irreducible component, and $\theta \in \mathbb{Z}^{Q_{0}}$ an integral weight of $A$. We say that $C$ is a $\theta$ (-semi)-stable irreducible component if $C$ contains a $\theta$ (-semi)stable $A$-module. A $\theta$-semistable irreducible component $C \subseteq \bmod (A, \boldsymbol{d})$ is said to be $\theta$-well-behaved if $\bmod \left(A, \boldsymbol{d}^{\prime}\right)$ has a unique $\theta$-stable irreducible component whenever $\boldsymbol{d}^{\prime}$ is the dimension vector of a factor of a Jordan-Hölder filtration in $\bmod (A)_{\theta}^{s s}$ of a generic $A$-module in $C$.

Example 3.3. If $A$ is a tame quasitilted algebra, then any $\theta$-semistable irreducible component is $\theta$-well-behaved. This is because for any generic $\operatorname{root} \boldsymbol{d}$ of $A$, as shown by Bobiński and Skowroński $[1999 \mathrm{~b}], \bmod (A, \boldsymbol{d})$ has a unique indecomposable irreducible component.

Let $C$ be a $\theta$-well-behaved irreducible component of $\bmod (A, \boldsymbol{d})$. We say that

$$
C=C_{1}+\cdots+C_{l}
$$

is the $\theta$-stable decomposition of $C$ if

- the $C_{i} \subseteq \bmod \left(A, \boldsymbol{d}_{i}\right)$ for $1 \leq i \leq l$ are $\theta$-stable irreducible components; and

- the generic $A$-module $M$ in $C$ has a finite filtration $0=M_{0} \subseteq M_{1} \subseteq \cdots \subseteq$ $M_{l}=M$ of submodules such that each factor $M_{j} / M_{j-1}$ for $1 \leq j \leq l$ is isomorphic to a $\theta$-stable module in one of the $C_{1}, \ldots, C_{l}$, and the sequence $\left(\operatorname{dim} M_{1} / M_{0}, \ldots, \operatorname{dim} M / M_{l-1}\right)$ is the same as $\left(d_{1}, \ldots, d_{l}\right)$ up to permutation.

To prove the existence and uniqueness of the $\theta$-stable decomposition of $C$, first note that the irreducible variety $C_{\theta}^{s s}$ is a disjoint union of sets of the form $\mathscr{F}_{(}\left(C_{i}\right)_{1 \leq i \leq l}$, where each $\mathscr{F}_{(}\left(C_{i}\right)_{1 \leq i \leq l}$ consists of those modules $M \in C$ that have a finite filtration $0=M_{0} \subseteq M_{1} \subseteq \cdots \subseteq M_{l}=M$ of submodules with each factor 
$M_{j} / M_{j-1}$ isomorphic to a $\theta$-stable module in one of the $C_{i}$ for $1 \leq i \leq l$. (Note that the $\theta$-well-behavedness of $C$ is used to ensure that the union above is indeed disjoint.) Next, it is not difficult to show that each $\mathscr{F}\left(C_{i}\right)_{1 \leq i \leq l}$ is constructible; see for example [Crawley-Boevey and Schröer 2002, Section 3]. Hence, there is a unique (up to permutation) sequence $\left(C_{i}\right)_{1 \leq i \leq l}$ of $\theta$-stable irreducible components for which $\mathscr{F}_{(}\left(C_{i}\right)_{1 \leq i \leq l}$ contains an open and dense subset of $C_{\theta}^{s S}$ (or $C$ ).

Remark 2. Let us mention that the notion of $\theta$-stable decomposition of a dimension vector in an irreducible component of a module variety was introduced in [Chindris 2011, Section 6.2]. It serves as a useful tool for finding convenient orthogonal exceptional sequences. But in order to understand how weight spaces of semiinvariants behave with respect to such a decomposition, one also needs to be able to keep track of the various $\theta$-stable irreducible components that arise in the decomposition in question. This issue is now addressed in the notion above of $\theta$-stable decomposition of a well-behaved irreducible component.

Next, we recall the following useful fact from invariant theory. Let $G$ and $G_{1}$ be linearly reductive groups with $G_{1} \leq G$, let $V$ be a finite-dimensional rational representation of $G$, and let $V_{1}$ be a vector subspace of $V$ invariant under the action of $G_{1}$. The $G_{1}$-equivariant inclusion $\tau: V_{1} \hookrightarrow V$ descends to a morphism

$$
\psi: V_{1} / / G_{1} \rightarrow V / / G
$$

such that $\psi \circ \pi_{1}=\pi \circ \tau$, where $\pi: V \rightarrow V / / G$ and $\pi_{1}: V_{1} \rightarrow V_{1} / / G_{1}$ are the categorical affine quotient morphisms. We denote the image of the zero vector of $V$ through the two quotient morphisms by the same symbol 0. Consider the Hilbert's nullcones $\mathcal{N}_{G}(V):=\pi^{-1}(0)$ and $\mathcal{N}_{G_{1}}\left(V_{1}\right):=\pi_{1}^{-1}(0)$.

Lemma 3.4. Keep the same notation as above. If $\psi^{-1}(0)=\{0\}$, then $\psi$ is a finite morphism.

Proof. Let $I$ be the ideal of $K[V]$ generated by all homogeneous $G$-invariants of positive degree. By choosing homogeneous invariants $f_{1}, \ldots, f_{n} \in K[V]^{G}$ such that $I=\left(f_{1}, \ldots, f_{n}\right)$, Hilbert proved that $K[V]^{G}=K\left[f_{1}, \ldots, f_{n}\right]$; see for example [Derksen and Kemper 2002, Theorem 2.2.10].

Now, if $\mathfrak{m}$ denotes the ideal of $K[V]^{G}$ generated by $f_{1}, \ldots f_{n}$, then the zero set of $\mathfrak{m}$ in $V / / G$ is precisely $\{0\}$. From this fact and the assumption that $\psi^{-1}(0)=\{0\}$, we immediately deduce that the zero set of $\psi^{*}\left(f_{1}\right), \ldots, \psi^{*}\left(f_{n}\right)$ in $V_{1}$ is precisely the nullcone $\mathcal{N}_{G_{1}}\left(V_{1}\right)$. Hence, $K\left[V_{1}\right]^{G_{1}}$ is a finite module over

$$
K\left[\psi^{*}\left(f_{1}\right), \ldots, \psi^{*}\left(f_{n}\right)\right]
$$

see for example [Derksen and Kemper 2002, Lemma 2.4.5]. The proof follows. 
With the right definition of $\theta$-stable decomposition, the proof of Theorem 1.4 is essentially the same as that of [Derksen and Weyman 2011, Theorem 3.20]. Nonetheless, we provide below a detailed proof for completeness. In what follows, if $C^{\prime}$ is a $\theta$-stable irreducible component that occurs in the $\theta$-stable decomposition of $C$ with multiplicity $m$, we denote $\underbrace{C^{\prime} \dot{+} C^{\prime}+\cdots \dot{+} C^{\prime}}_{m}$ by $m \cdot C^{\prime}$.

Proof of Theorem 1.4. Without loss of generality, we assume that $\theta$ is indivisible, the induced character $\chi_{\theta} \in X^{*}(\mathrm{GL}(\boldsymbol{d}))$ is not trivial, and $Q$ is connected.

We view $\mathscr{V}$ as a vector subspace of $\bmod (Q, \boldsymbol{d})$ and denote by $G$ the stabilizer of $\mathscr{V} \subseteq \bmod (Q, \boldsymbol{d})$ in $G_{\theta}$. It easy to see that $G$ is isomorphic to the intersection of $G_{\theta}$ with

$$
\left(S_{m_{1}} \ltimes \mathrm{GL}\left(\boldsymbol{d}_{1}\right)^{m_{1}}\right) \times \cdots \times\left(S_{m_{n}} \ltimes \mathrm{GL}\left(\boldsymbol{d}_{n}\right)^{m_{n}}\right) .
$$

(Here, $S_{m}$ denotes the symmetric group on $m$ elements.) Let

$$
\psi: \mathscr{V} / / G \rightarrow \bmod (Q, \boldsymbol{d}) / / G_{\theta}
$$

be the morphism induced by the $G$-equivariant inclusion $\tau: \mathscr{V} \hookrightarrow \bmod (Q, \boldsymbol{d})$. Since $X$ embeds $G$-equivariantly into $C, \psi$ descends to a morphism

$$
\tau: X / / G \rightarrow C / / G_{\theta}
$$

such that $\tilde{\psi} \circ \pi_{X}=\left.\pi_{C} \circ \tau\right|_{X}$, where $\pi_{X}: X \rightarrow X / / G$ and $\pi_{C}: C \rightarrow C / / G_{\theta}$ are the categorical quotient morphisms. Note that

$$
K\left[C / / G_{\theta}\right]=\bigoplus_{m \geq 0} \operatorname{SI}(C)_{m \theta}, \quad \text { and } \quad K[X / / G]=\bigoplus_{m \geq 0} \bigotimes_{i=1}^{n} S^{m_{i}}\left(\operatorname{SI}\left(C_{i}\right)_{m \theta}\right),
$$

and moreover, the pullback map $\widetilde{\psi}^{*}$ respects the gradings of the coordinate rings above. In what follows we show that $\widetilde{\psi}^{*}$ is an isomorphism.

Note that if $M \in \mathscr{V}$, then $M$ is $G$-semistable, meaning that $0 \in \overline{G M}$ if and only if the direct summands of $M$ are $\theta$-semistable. This implies that $\psi^{-1}(0)=\{0\}$, and so $\psi$ is a finite morphism by Lemma 3.4. But since $\widetilde{\psi}$ is the restriction of $\psi$ to $X / / G$, we can immediately see that $\widetilde{\psi}$ is a finite morphism too.

Next, let $M \in C_{\theta}^{s s}$ be a module that has a filtration of the form

$$
0=M_{0} \subseteq M_{1} \subseteq \cdots \subseteq M_{l}=M,
$$

where the factors $M_{i} / M_{i-1}$ for $1 \leq i \leq l$ are $\theta$-stable and the sequence

$$
\left(\operatorname{dim} M_{1}, \ldots, \operatorname{dim} M / M_{l-1}\right)
$$

is the same as $\left(\boldsymbol{d}_{1}^{m_{1}}, \ldots, \boldsymbol{d}_{n}^{m_{n}}\right)$ up to permutation. Here, $l:=m_{1}+\cdots+m_{n}$. Now, let $\tilde{M} \in X$ be a module isomorphic to $\bigoplus_{i=1}^{l} M_{i} / M_{i-1}$. Then, we have

$$
\widetilde{\psi}\left(\pi_{X}(\tilde{M})\right)=\pi_{C}(M),
$$


and hence $\widetilde{\psi}$ is dominant. Denote by $X^{0}$ the nonempty open subset

$$
\left(C_{1, \theta}^{s}\right)^{m_{1}} \times \cdots \times\left(C_{n, \theta}^{s}\right)^{m_{n}}
$$

of $X$, and note that any point of $X^{0}$ has its $G_{\theta}$-orbit closed in $C$. This implies that $\pi_{C}$ is injective on $X^{0}$, and so the morphism $\widetilde{\psi}$ is injective on $\pi_{X}\left(X^{0}\right)$; in particular, $\widetilde{\psi}$ is injective on an open and dense subset of $X / / G$. It is now clear that $\widetilde{\psi}$ has to be a birational morphism.

Finally, we know from geometric invariant theory that the affine quotient variety $C / / G_{\theta}$ is normal, since $C$ is assumed to be a normal variety. It now follows that $\widetilde{\psi}$ is an isomorphism, and this finishes the proof.

Remark 3. Keep the same assumptions as in Theorem 1.4. If we further assume that $A$ is tame, then for each $1 \leq i \leq n$, the moduli space $M\left(C_{i}\right)_{\theta}^{s S}$ is of dimension $\operatorname{dim} C_{i}-\operatorname{dim} \operatorname{GL}\left(\boldsymbol{d}_{i}\right)+1 \leq 1$. More precisely, $\mathcal{M}\left(C_{i}\right)_{\theta}^{s s}$ is a curve if, for example, $q_{A}\left(\boldsymbol{d}_{i}\right)=0$; see [de la Peña 1991, Proposition 1.2].

Hence, the "building blocks" $\mathcal{M}\left(C_{1}\right)_{\theta}^{s s}, \ldots, \mathcal{M}\left(C_{n}\right)_{\theta}^{s s}$ that make up the moduli space $\mathcal{M}(C)_{\theta}^{s s}$ are either points or projective curves in the tame case.

\section{Tilted algebras}

Recall that a quasitilted algebra is a basic and connected finite-dimensional algebra of the form $\operatorname{End}_{\mathscr{H}}(T)^{\mathrm{op}}$, where $\mathscr{H}$ is a hereditary category and $T \in \mathscr{H}$ is a tilting object.

\section{A. Singular moduli spaces of modules for wild tilted algebras. Let}

$$
B=\operatorname{End}_{A}(T)^{\mathrm{op}}
$$

be a wild tilted algebra, where $A=k Q$ with $Q$ a wild connected quiver and $T$ is a basic tilting $A$-module. Our goal here is to show that $B$ has a singular moduli space of modules. We achieve this by reducing the considerations to the case of wild hereditary algebras via Theorem 1.3.

Proposition 4.1. If $B$ is a wild tilted algebra, then there exist a generic root $\boldsymbol{d}$ of $B$, an indecomposable irreducible component $C$ of $\bmod (B, \boldsymbol{d})$, and an integral weight $\theta$ of $B$ such that $C_{\theta}^{s} \neq \varnothing$ and the moduli space $\mathcal{M}(C)_{\theta}^{s s}$ is singular.

Proof. First of all, we know from the main results in [Kerner 1989; 1997] and [Strauss 1991] that any wild tilted algebra contains a convex subcategory that is wild concealed (the titling module involved is either preprojective or preinjective). Consequently, we can assume that $B=\operatorname{End}_{A}(T)^{\text {op }}$, where $A=k Q$ with $Q$ a connected wild quiver and $T$ is a basic preprojective tilting $A$-module. (The case when $T$ is preinjective is dual.) Then, we know that the indecomposable $A$-modules in 
$\mathscr{F}(T)$ are all preprojective and any regular or preinjective $A$-module belongs to $\mathscr{T}(T)$; see for example [Assem et al. 2006].

To construct a weight $\theta$ with the desired properties, we begin by choosing a regular $A$-module $X_{0}$ with the property that all $\tau_{A}^{m} X$ for $m \geq 0$ are sincere regular Schur $A$-modules and $\operatorname{dim} X_{0}$ is an imaginary, nonisotropic root of $A$; see [Kerner 1996, Proposition 10.2]. Denote the dimension vector of $X_{0}$ by $\boldsymbol{d}_{0}$ and let $\theta_{0}$ be the weight $\left\langle\boldsymbol{d}_{0}, \cdot\right\rangle_{A}-\left\langle\cdot, \boldsymbol{d}_{0}\right\rangle_{A}$. Then $n \boldsymbol{d}_{0}$ is $\theta_{0}$-stable for all integers $n \in \mathbb{Z}_{>0}$ by [Schofield 1992, Theorem 6.1] and [Derksen and Weyman 2011, Proposition 3.16].

Next, we show that $\theta_{0}$ is well positioned with respect to $T$, which is equivalent to showing that $\theta_{0}(\operatorname{dim} M)<0$ for every preprojective $A$-module $M$. Assume to the contrary that there exists a preprojective $A$-module $M \operatorname{such}$ that $\langle\operatorname{dim} X, \operatorname{dim} M\rangle \geq$ $\langle\operatorname{dim} M, \operatorname{dim} X\rangle$. But this is equivalent to

$$
-\operatorname{dim}_{k} \operatorname{Ext}_{A}^{1}(X, M) \geq \operatorname{dim}_{k} \operatorname{Hom}_{A}(M, X),
$$

and so $\operatorname{dim}_{k} \operatorname{Ext}_{A}^{1}(X, M)=0$. Writing $M=\tau_{A}^{-m} P_{i}$ for uniquely determined $m \in \mathbb{Z}_{\geq 0}$ and $i \in Q_{0}$, we get that $\tau_{A}^{m+1} X(i)=\{0\}$, which contradicts that $\tau_{A}^{m+1} X$ is sincere. So, we conclude that $\theta_{0}$ is well positioned with respect to $T$.

Let $u: K_{0}(A) \rightarrow K_{0}(B)$ be the isometry induced by $T$ and let $\theta=\theta_{0} \circ u^{-1}$. We claim that $C:=\overline{\bmod (B, \boldsymbol{d})_{\theta}^{s s}}$ is an irreducible component of $\bmod (B, \boldsymbol{d})$, where $\boldsymbol{d}:=u\left(n \boldsymbol{d}_{0}\right)$ and $n \in \mathbb{Z}_{>0}$. Indeed, it follows from the proof of Theorem 1.3(a) that the $\theta$-semistable $B$-modules all lie in $\mathscr{Y}(T)$, and hence their projective dimension is at most one, as $A$ is hereditary. Consequently, the $\operatorname{subset} \bmod \mathscr{P}(B, \boldsymbol{d}) \operatorname{of} \bmod (B, \boldsymbol{d})$ consisting of all modules of projective dimension at most one is nonempty, and this implies that $\bmod _{\mathscr{P}}(B, \boldsymbol{d})$ is an irreducible open subset of $\bmod (B, \boldsymbol{d})$; see [Barot and Schröer 2001, Proposition 3.1]. This immediately implies our claim. Furthermore, as $n \boldsymbol{d}_{0}$ is $\theta_{0}$-stable, we deduce from the proof of Theorem 1.3(a) that $\boldsymbol{d}$ is $\theta$-stable, that is, $C_{\theta}^{s} \neq \varnothing$.

Using Theorem 1.3(b) again, we get that $\mathcal{M}(C)_{\theta}^{s s} \simeq \mathcal{M}\left(A, n \boldsymbol{d}_{0}\right)_{\theta_{0}}^{s s}$, which is known to be singular for $n=3$; see for example [Domokos 2011].

Proof of Proposition 1.2. Assuming to the contrary that $A$ is wild, it follows from [Brüstle et al. 2011, Corollary 1] that $A$ contains a convex hypercritical algebra $B$. Then Proposition 4.1 provides us with a singular moduli space of $B$-modules, which contradicts our assumption on the moduli spaces of modules for $A$.

Remark 4. In [Brüstle et al. 2011], Brüstle, de la Peña, and Skowroński proved that for a tame strongly simply connected algebra $A$, the convex hull of any indecomposable $A$-module inside $A$ is a tame tilted algebra, or a coil algebra, or a $\mathbb{D}$-algebra; see [Brüstle et al. 2011, Corollary 5]. Hence, to prove the analogue of Theorem 1.1 for strongly simply connected algebras, which was conjectured 
to hold true by Weyman, it remains to study the geometry of modules over coil algebras and $\mathbb{D}$-algebras. We plan to address these issues in future work.

\section{B. Rational and GIT quotient varieties of modules for tame quasitilted alge-}

bras. In what follows, we review some important facts about the geometry of modules over quasitilted algebras, which are due to Bobiński and Skowroński.

By a root of a quasitilted algebra $A$, we simply mean the dimension vector of an indecomposable $A$-module. We say that a root $\boldsymbol{d}$ of $A$ is real if $q_{A}(\boldsymbol{d})=1$. We call a root $\boldsymbol{d}$ of $A$ isotropic if $q_{A}(\boldsymbol{d})=0$. If $\boldsymbol{d}$ is an isotropic generic root of $A$, we call the indecomposable irreducible components of $\bmod (A, \boldsymbol{d})$ isotropic, too.

Now, we can state the following important result; see [Bobiński and Skowroński 1999b, Corollaries 3 and 2.5 and Proposition 2.3].

Theorem 4.2. Let $A$ be a tame quasitilted algebra and let $\boldsymbol{d}$ be a generic root of $A$. Then $\boldsymbol{d}$ is a Schur root with $q_{A}(\boldsymbol{d}) \in\{0,1\}$. More precisely:

(1) If $q_{A}(\boldsymbol{d})=1$, there exists a unique, up to isomorphism, $\boldsymbol{d}$-dimensional indecomposable A-module $M$ that is, in fact, exceptional; if this is the case, then $\overline{\mathrm{GL}}(\boldsymbol{d}) M$ is the unique indecomposable irreducible component of $\bmod (A, \boldsymbol{d})$.

(2) If $q_{A}(\boldsymbol{d})=0$, the support of $\boldsymbol{d}$ is a tame concealed or a tubular convex subcategory of $A$. Furthermore, $\bmod (A, \boldsymbol{d})$ is a normal variety.

Proposition 4.3 [Chindris 2011]. Let A be a tame concealed or a tubular algebra, and $\boldsymbol{d}$ an isotropic Schur root of $A$. Then there exists a short orthogonal exceptional sequence $\mathscr{E}=\left(E_{1}, E_{2}\right)$ with $\operatorname{dim}_{k} \operatorname{Ext}_{A}^{1}\left(E_{2}, E_{1}\right)=2$ and $\operatorname{Ext}_{A}^{2}\left(E_{2}, E_{1}\right)=0$, and such that the generic module $M$ in $\bmod (A, d)$ fits into a short exact sequence of the form

$$
0 \longrightarrow E_{1} \longrightarrow M \longrightarrow E_{2} \longrightarrow 0 \text {. }
$$

Remark 5. This proposition has been proved for tame canonical algebras in [Chindris 2011, Proposition 6.7], but the exact same arguments work for arbitrary tame concealed algebras and for tubular algebras; see for example [Chindris 2012].

Proposition 4.4. Let A be a quasitilted algebra.

(1) The following conditions are equivalent:

(a) A is tame;

(b) for each generic root $\boldsymbol{d}$ of $A$ and each indecomposable irreducible component $C$ of $\bmod (A, \boldsymbol{d})$, either $k(C)^{\mathrm{GL}(\boldsymbol{d})} \simeq k$ or $k(x)$.

(2) Assume $A$ is tame and let $\boldsymbol{d}$ be an isotropic root of $A$. Then $\mathcal{M}(\bmod (A, \boldsymbol{d}))_{\theta}^{s s}$ is a product of projective spaces for every integral weight $\theta$ of $A$.

Proof. (1) The implication (b) $\Longrightarrow$ (a) has been already proved in [Chindris 2011, Proposition 4.6]. 
Now, let us assume that $A$ is tame and let $\boldsymbol{d}$ be a generic root of $A$. We know from Theorem 4.2 that $\boldsymbol{d}$ is a $S$ chur root and $\bmod (A, \boldsymbol{d})$ has a unique indecomposable irreducible component; call it $C$.

If $q_{A}(\boldsymbol{d})=1$, then $k(C)^{\mathrm{GL}(\boldsymbol{d})} \simeq k$ since $C$ is just the closure of the $\mathrm{GL}(\boldsymbol{d})$-orbit of the $\boldsymbol{d}$-dimensional exceptional $A$-module.

It remains to look into the case when $\boldsymbol{d}$ is an isotropic Schur root of $A$. In this case, we simply use Proposition 4.3 and Proposition 2.2 to conclude that $k(C)^{\mathrm{GL}(d)} \simeq k(x)$.

(2) We know that $\bmod (A, \boldsymbol{d})$ is normal by Corollary 3 in [Bobiński and Skowroński 1999b]. Now, let $\theta$ be an integral weight for which $\mathcal{M}(A, \boldsymbol{d})_{\theta}^{s s} \neq \varnothing$, and note that $\bmod (A, \boldsymbol{d})$ is $\theta$-well-behaved by Theorem 4.2. Let $C_{1}, \ldots, C_{n}$ be the pairwise distinct isotropic indecomposable irreducible components that occur in the $\theta$-stable decomposition of $\bmod (A, \boldsymbol{d})$, and denote by $m_{1}, \ldots, m_{n}$ their multiplicities. It now follows from Theorem 1.4 that

$$
\mu(A, \boldsymbol{d})_{\theta}^{s s} \cong \prod_{i=1}^{n} S^{m_{i}}\left(\mathcal{M}\left(C_{i}\right)_{\theta}^{s s}\right) .
$$

But, for each $1 \leq i \leq n, \mathcal{M}\left(C_{i}\right)_{\theta}^{s s}$ is a projective curve that is, first, normal, as $C_{i}$ is normal by Theorem 4.2(2) and, second, rational, as proved in part (1). So, $\mathcal{M}\left(C_{i}\right)_{\theta}^{S S} \simeq \mathbb{P}^{1}$ for all $1 \leq i \leq n$, and hence $\mathcal{M}(A, \boldsymbol{d})_{\theta}^{S S} \cong \prod_{i=1}^{n} \mathbb{P}^{m_{i}}$.

Remark 6. Let $A$ be a tame quasitilted algebra, $\boldsymbol{d}$ a root of $A, C \subseteq \bmod (A, \boldsymbol{d})$ an irreducible component, and $\theta$ an integral weight of $A$ such that $C_{\theta}^{s} \neq \varnothing$. Then the proposition above tells us that $\mathcal{M}(C)_{\theta}^{s s}$ is either a point or just $\mathbb{P}^{1}$.

Proof of Theorem 1.1. The implications (1) $\Longrightarrow$ (2) $\Longrightarrow$ (3) $\Longrightarrow$ (4) were proved in Proposition 4.4. The implication (4) $\Longrightarrow$ (1) follows from Proposition 4.1.

Proof of Proposition 1.5. We know from Theorem 1.1 that if $C$ is an indecomposable irreducible component of $\bmod (A, \boldsymbol{d})$, then $S^{m}\left(k(C)^{\mathrm{GL}(\boldsymbol{d})}\right)$ is isomorphic to either $k$, in case $\boldsymbol{d}$ is a real Schur root, or $k\left(t_{1}, \ldots, t_{m}\right)$, in case $\boldsymbol{d}$ is isotropic. The proof now follows from Proposition 2.1 and Proposition 4.4.

Remark 7. In view of [Happel 2001], to prove the implication (4) $\Rightarrow$ (1) of Theorem 1.1 for quasitilted algebras, one possible path is to prove first the analogue of Theorem 1.3 for tilting complexes, and then that of Proposition 4.1 for wild canonical algebras. We plan to explore this approach in a sequel to this work.

\section{Acknowledgments}

I am grateful to Otto Kerner for clarifying conversations on wild tilted algebras. I would also like to thank the referee for detailed suggestions that helped improve the exposition of the paper. 


\section{References}

[Assem et al. 2006] I. Assem, D. Simson, and A. Skowroński, Elements of the representation theory of associative algebras, 1: Techniques of representation theory, London Mathematical Society Student Texts 65, Cambridge University Press, 2006. MR 2006j:16020 Zbl 1092.16001

[Barot and Schröer 2001] M. Barot and J. Schröer, "Module varieties over canonical algebras", J. Algebra 246:1 (2001), 175-192. MR 2003e:16013 Zbl 1036.16010

[Bobiński 2008] G. Bobiński, "On the zero set of semi-invariants for regular modules over tame canonical algebras”, J. Pure Appl. Algebra 212:6 (2008), 1457-1471. MR 2009c:16042 Zbl 1180. 16011

[Bobiński and Skowroński 1999a] G. Bobiński and A. Skowroński, "Geometry of directing modules over tame algebras", J. Algebra 215:2 (1999), 603-643. MR 2000f:16017 Zbl 0965.16009

[Bobiński and Skowroński 1999b] G. Bobiński and A. Skowroński, "Geometry of modules over tame quasi-tilted algebras", Colloq. Math. 79:1 (1999), 85-118. MR 2000i:14067 Zbl 0994.16009

[Bobiński and Skowroński 2002] G. Bobiński and A. Skowroński, "Geometry of periodic modules over tame concealed and tubular algebras", Algebr. Represent. Theory 5:2 (2002), 187-200. MR 2003d:16021 Zbl 1013.16009

[Bongartz 1983] K. Bongartz, "Algebras and quadratic forms", J. London Math. Soc. (2) 28:3 (1983), 461-469. MR 85i:16036 Zbl 0532.16020

[Brüstle et al. 2011] T. Brüstle, J. A. de la Peña, and A. Skowroński, "Tame algebras and Tits quadratic forms", Adv. Math. 226:1 (2011), 887-951. MR 2011m:16023 Zbl 05825430

[Chindris 2011] C. Chindris, "Geometric characterizations of the representation type of hereditary algebras and of canonical algebras", Adv. Math. 228:3 (2011), 1405-1434. MR 2012h:16033 Zbl 05949109

[Chindris 2012] C. Chindris, "On the geometry of orbit closures for representation-infinite algebras", Glasg. Math. J. 54:3 (2012), 629-636.

[Crawley-Boevey and Schröer 2002] W. Crawley-Boevey and J. Schröer, "Irreducible components of varieties of modules", J. Reine Angew. Math. 553 (2002), 201-220. MR 2004a:16020 Zbl 1062. 16019

[Derksen and Kemper 2002] H. Derksen and G. Kemper, Computational invariant theory, Encyclopaedia of Mathematical Sciences 130, Springer, Berlin, 2002. MR 2003g:13004 Zbl 1011.13003

[Derksen and Weyman 2011] H. Derksen and J. Weyman, "The combinatorics of quiver representations”, Ann. Inst. Fourier (Grenoble) 61:3 (2011), 1061-1131. MR 2918725 Zbl 06002991

[Domokos 2011] M. Domokos, "On singularities of quiver moduli”, Glasg. Math. J. 53:1 (2011), 131-139. MR 2012a:16029 Zbl 1241.16010

[Domokos and Lenzing 2000] M. Domokos and H. Lenzing, "Invariant theory of canonical algebras”, J. Algebra 228:2 (2000), 738-762. MR 2001h:16016 Zbl 0955.16015

[Domokos and Lenzing 2002] M. Domokos and H. Lenzing, "Moduli spaces for representations of concealed-canonical algebras", J. Algebra 251:1 (2002), 371-394. MR 2003d:16016 Zbl 1013. 16006

[Drozd 1979] J. A. Drozd, "Tame and wild matrix problems", pp. 39-74 in Representations and quadratic forms, edited by Y. A. Mitropol'skiı̌, Akad. Nauk Ukrain. SSR Inst. Mat., Kiev, 1979. In Russian; translated in Amer. Math. Soc. Transl. Ser. 2128 (1986), 31-55. MR 82m:16028 Zbl 0454.16014

[Geiss and Schröer 2003] C. Geiss and J. Schröer, "Varieties of modules over tubular algebras", Colloq. Math. 95:2 (2003), 163-183. MR 2004d:16026 Zbl 1033.16004

[Happel 2001] D. Happel, "A characterization of hereditary categories with tilting object", Invent. Math. 144:2 (2001), 381-398. MR 2002a:18014 Zbl 1015.18006 
[Happel et al. 1996] D. Happel, I. Reiten, and S. O., Tilting in abelian categories and quasitilted algebras, Mem. Amer. Math. Soc. 120, 1996. MR 97j:16009 Zbl 0849.16011

[Kerner 1989] O. Kerner, "Tilting wild algebras", J. London Math. Soc. (2) 39:1 (1989), 29-47. MR 90d:16025 Zbl 0675.16013

[Kerner 1996] O. Kerner, "Representations of wild quivers", pp. 65-107 in Representation theory of algebras and related topics (Mexico City, 1994), edited by R. Bautista et al., CMS Conf. Proc. 19, Amer. Mathematical Society, Providence, RI, 1996. MR 97e:16028 Zbl 0863.16010

[Kerner 1997] O. Kerner, "Wild tilted algebras revisited", Colloq. Math. 73:1 (1997), 67-81. MR 98b:16011 Zbl 0879.16006

[King 1994] A. D. King, "Moduli of representations of finite-dimensional algebras", Quart. J. Math. Oxford Ser. (2) 45:180 (1994), 515-530. MR 96a:16009 Zbl 0837.16005

[Kraśkiewicz 2001] W. Kraśkiewicz, "On semi-invariants of tilted algebras of type $A_{n}$ ", Colloq. Math. 90:2 (2001), 253-267. MR 2002m:16015 Zbl 0993.16010

[Le Potier 1997] J. Le Potier, Lectures on vector bundles, Cambridge Studies in Advanced Mathematics 54, Cambridge University Press, 1997. MR 98a:14019 Zbl 0872.14003

[de la Peña 1991] J. A. de la Peña, "On the dimension of the module-varieties of tame and wild algebras”, Comm. Algebra 19:6 (1991), 1795-1807. MR 92i:16016 Zbl 0818.16013

[Riedtmann 2004] C. Riedtmann, “Tame quivers, semi-invariants, and complete intersections”, $J$. Algebra 279:1 (2004), 362-382. MR 2005j:16015 Zbl 1076.16013

[Riedtmann and Zwara 2004] C. Riedtmann and G. Zwara, "On the zero set of semi-invariants for tame quivers", Comment. Math. Helv. 79:2 (2004), 350-361. MR 2005g:16024 Zbl 1063.14052

[Riedtmann and Zwara 2008] C. Riedtmann and G. Zwara, "The zero set of semi-invariants for extended Dynkin quivers”, Trans. Amer. Math. Soc. 360:12 (2008), 6251-6267. MR 2009i:14064 Zbl 1159.14306

[Schofield 1992] A. Schofield, "General representations of quivers", Proc. London Math. Soc. (3) 65:1 (1992), 46-64. MR 93d:16014 Zbl 0795.16008

[Simson and Skowroński 2007] D. Simson and A. Skowroński, Elements of the representation theory of associative algebras, 3: Representation-infinite tilted algebras, London Mathematical Society Student Texts 72, Cambridge University Press, 2007. MR 2008m:16001 Zbl 1131.16001

[Skowroński 1993] A. Skowroński, "Simply connected algebras and Hochschild cohomologies [ MR1206961 (94e:16016)]", pp. 431-447 in Representations of algebras (Ottawa, 1992), CMS Conf. Proc. 14, American Mathematical Society, Providence, RI, 1993. MR 1265301 Zbl 0806. 16012

[Skowroński 1998] A. Skowroński, “Tame quasi-tilted algebras”, J. Algebra 203:2 (1998), 470-490. MR 99b:16019 Zbl 0908.16013

[Skowroński and Weyman 2000] A. Skowroński and J. Weyman, "The algebras of semi-invariants of quivers", Transform. Groups 5:4 (2000), 361-402. MR 2001m:16017 Zbl 0986.16004

[Strauss 1991] H. Strauss, "On the perpendicular category of a partial tilting module", J. Algebra 144:1 (1991), 43-66. MR 92m:16013 Zbl 0746.16009

[Weibel 1994] C. A. Weibel, An introduction to homological algebra, Cambridge Studies in Advanced Mathematics 38, Cambridge University Press, 1994. MR 95f:18001 Zbl 0797.18001

Communicated by J. Toby Stafford

Received 2011-09-17 Revised 2012-01-02 Accepted 2012-02-20

chindrisc@missouri.edu Department of Mathematics, University of Missouri, Columbia, MO 65211, United States http://www.math.missouri.edu/ chindrisc 


\section{Algebra \& Number Theory}

msp.org/ant

\section{EDITORS}

MANAGING EDITOR

Bjorn Poonen

Massachusetts Institute of Technology

Cambridge, USA

\author{
EDITORIAL BOARD CHAIR \\ David Eisenbud \\ University of California \\ Berkeley, USA
}

\section{BOARD OF EDITORS}

Georgia Benkart

Dave Benson

Richard E. Borcherds

John H. Coates

J-L. Colliot-Thélène

Brian D. Conrad

Hélène Esnault

Hubert Flenner

Edward Frenkel

Andrew Granville

Joseph Gubeladze

Ehud Hrushovski

Craig Huneke

Mikhail Kapranov

Yujiro Kawamata

János Kollár

Yuri Manin

Barry Mazur

Philippe Michel
University of Wisconsin, Madison, USA

University of Aberdeen, Scotland

University of California, Berkeley, USA

University of Cambridge, UK

CNRS, Université Paris-Sud, France

University of Michigan, USA

Freie Universität Berlin, Germany

Ruhr-Universität, Germany

University of California, Berkeley, USA

Université de Montréal, Canada

San Francisco State University, USA

Hebrew University, Israel

University of Virginia, USA

Yale University, USA

University of Tokyo, Japan

Princeton University, USA

Northwestern University, USA

Harvard University, USA

École Polytechnique Fédérale de Lausanne
Susan Montgomery

Shigefumi Mori

Raman Parimala

Jonathan Pila

Victor Reiner

Karl Rubin

Peter Sarnak

Joseph H. Silverman

Michael Singer

Vasudevan Srinivas

J. Toby Stafford

Bernd Sturmfels

Richard Taylor

Ravi Vakil

Michel van den Bergh

Marie-France Vignéras

Kei-Ichi Watanabe

Efim Zelmanov
University of Southern California, USA

RIMS, Kyoto University, Japan

Emory University, USA

University of Oxford, UK

University of Minnesota, USA

University of California, Irvine, USA

Princeton University, USA

Brown University, USA

North Carolina State University, USA

Tata Inst. of Fund. Research, India

University of Michigan, USA

University of California, Berkeley, USA

Harvard University, USA

Stanford University, USA

Hasselt University, Belgium

Université Paris VII, France

Nihon University, Japan

University of California, San Diego, USA

\section{PRODUCTION}

production@msp.org

Silvio Levy, Scientific Editor

See inside back cover or msp.org/ant for submission instructions.

The subscription price for 2013 is US \$200/year for the electronic version, and \$350/year ( $\$ 40$, if shipping outside the US) for print and electronic. Subscriptions, requests for back issues and changes of subscribers address should be sent to MSP.

Algebra \& Number Theory (ISSN 1944-7833 electronic, 1937-0652 printed) at Mathematical Sciences Publishers, 798 Evans Hall \#3840, c/o University of California, Berkeley, CA 94720-3840 is published continuously online. Periodical rate postage paid at Berkeley, CA 94704, and additional mailing offices.

ANT peer review and production are managed by EditFLOW ${ }^{\circledR}$ from Mathematical Sciences Publishers.

\section{PUBLISHED BY}

mathematical sciences publishers

nonprofit scientific publishing

http://msp.org/

(C) 2013 Mathematical Sciences Publishers 


\section{Algebra \& Number Theory}

\section{Volume $7 \quad$ No. $1 \quad 2013$}

Powers of ideals and the cohomology of stalks and fibers of morphisms

MARC CHARDIN

Graphs of Hecke operators

OLIVER LORSCHEID

Group actions of prime order on local normal rings

FrANZ KIRÀLY and WERNER LÜTKEBOHMERT

On the arithmetic and geometry of binary Hamiltonian forms

JOUNI PARKKONEN and FRÉDÉRIC PAULIN

$L$-functions and periods of adjoint motives

MICHAEL HARRIS

Galois module structure of local unit groups

ROMYAR SHARIFI

On the invariant theory for tame tilted algebras

CALIN CHINDRIS

Period functions and cotangent sums

SANDRO BETTIN and BRIAN CONREY 